\title{
TREND TO STEADY STATE IN A CONSERVATION LAW WITH SPATIAL INHOMOGENEITY*
}

\author{
BY \\ C. M. DAFERMOS \\ Lefschetz Center for Dynamical Systems, Brown University
}

1. Introduction. The writing of this note was motivated by [7]; in that paper the authors seek to model the steady, choked, isentropic flow of a gas in a dual-throat nozzle with equal throat areas as an initial-boundary value problem for a conservation law with spatial inhomogeneity:

$$
\begin{gathered}
\partial_{t} u(x, t)+\partial_{x} f(u(x, t), x)=0, \quad 0<x<1, \quad 0<t<\infty, \\
u(x, 0)=\phi(x), \quad 0<x<1, \\
\left\{\begin{array}{l}
f_{u}(u(0+, t), 0) \leqslant 0 \\
f_{u}(u(1-, t), 1) \geqslant 0
\end{array} \quad 0<t<\infty .\right.
\end{gathered}
$$

Here $\phi$ is a given bounded measurable function on $(0,1)$ and $f$ is a $C^{2}$ function on $(-\infty, \infty) \times(-\infty, \infty)$ which satisfies the following conditions:

$$
\begin{gathered}
f_{u}(0, x)=0, \quad-\infty<x<\infty, \\
f_{u u}(u, x)>0, \quad-\infty<u<\infty, \quad-\infty<x<\infty, \\
f(u, x+1)=f(u, x), \quad-\infty<u<\infty, \quad-\infty<x<\infty, \\
f(0,0)=0, \\
\begin{cases}f_{x}(0, x)<0, & 0<x<a \\
f_{x}(0, x)>0, & a<x<1,\end{cases}
\end{gathered}
$$

for some $a \in(0,1)$.

\footnotetext{
* Received April 10, 1986.

${ }^{1}$ This research was supported by the National Science Foundation under Grant No. DMS-8205355, by the U.S. Army under Contract No. DAAG-29-83-K-0029, and by the Office of Naval Research under Contract No. N00014-83-K-0542.
} 
One of the specific models proposed in [7] is

$$
f(u, x):=u^{2}-\sin ^{2}(\pi x)
$$

Note that the role of the boundary conditions $(1.3)$ is to insulate the interval $(0,1)$ from external influence; they allow (and in fact, as we shall see, often induce) the boundaries to become characteristic. In Section 3 we will show that the problem (1.1), (1.2), (1.3) is well posed.

Equation (1.1) admits two smooth steady state solutions compatible with the boundary conditions $(1,3)$, one completely supersonic, denoted by $v_{+}(x)$, and one completely subsonic, denoted by $v_{-}(x)$. In addition, there are infinitely many admissible discontinuous steady state solutions with shock waves. It was shown in [7] that, as $t \rightarrow \infty$, the solution of (1.1), (1.2), (1.3) will converge to the supersonic steady state solution $v_{+}(x)$ or to the subsonic steady state solution $v_{-}(x)$ or to a discontinuous steady state solution with a single shock that connects the supersonic to the subsonic branch. It is interesting to determine the precise form of the asymptotic profile in terms of the initial datum $\phi$. This was indeed attained in [7], albeit for rather special $\phi$. Our objective here is to demonstrate that the method of generalized characteristics (cf. [1, 2, 3, 4]) determines, with accuracy, the large time behavior of solutions, under general initial data $\phi$.

I am indebted to David Gottlieb for helpful discussions.

2. Classical characteristics. A (classical) characteristic for (1.1), associated with a particular Lipschitz solution $u(x, t)$, is an integral curve $\xi(\cdot)$ of the ordinary differential equation

$$
\dot{\xi}(t)=f_{u}(u(\xi(t), t), \xi(t))
$$

The restriction $w(t):=u(\xi(t), t)$ of $u$ on $\xi(\cdot)$ satisfies

$$
\dot{w}(t)=-f_{x}(u(\xi(t), t), \xi(t)) .
$$

Therefore, the family of characteristics for (1.1) is determined by integrating the system

$$
\left\{\begin{array}{l}
\dot{\xi}=f_{u}(w, \xi) \\
\dot{w}=-f_{x}(w, \xi)
\end{array}\right.
$$

It follows from (2.3) that along any characteristic

$$
\dot{f}(w, \xi)=0
$$

and so, recalling the properties (1.4)-(1.8) of $f(u, x)$, we deduce that the phase portrait of (2.3) is of the form depicted in Fig. 1. In particular, the restriction of the phase portrait over the strip $[0,1] \times(-\infty, \infty)$ contains a center, at the point $(a, 0)$, surrounded by periodic orbits, and two saddles, at $(0,0)$ and $(1,0)$, which are connected by heteroclinic orbits $w=v_{+}(\xi)$ and $w=v_{-}(\xi)$ determined as solutions to

$$
\left\{\begin{array}{lll}
f\left(v_{+}(\xi), \xi\right)=0, & v_{+}(\xi)>0, & 0<\xi<1 \\
f\left(v_{-}(\xi), \xi\right)=0, & v_{-}(\xi)<0, & 0<\xi<1 .
\end{array}\right.
$$




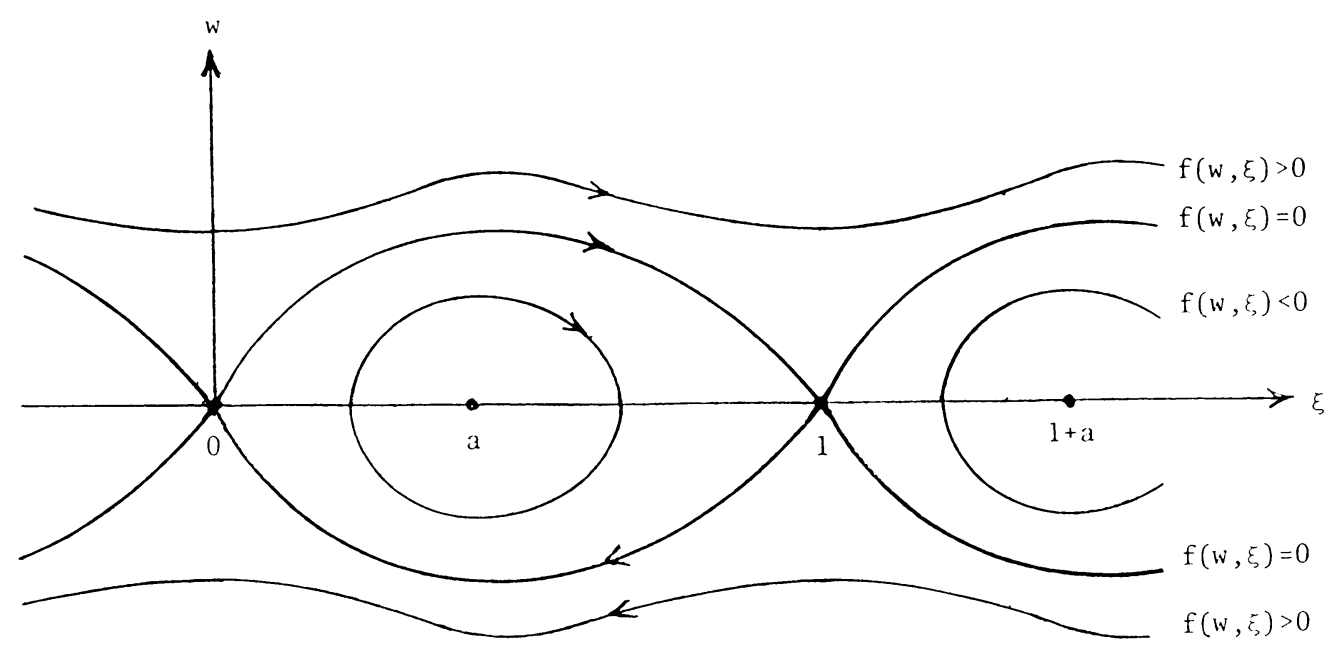

FIG. 1.

The orbit $w=v(\xi)$ of any characteristic induces a smooth steady state solution $u=v(x)$ of (1.1). The steady state solutions $u=v_{+}(x)$ and $u=v_{-}(x)$ are the only ones that are defined over $(0,1)$ and are compatible with the boundary conditions $(1.3)$.

In the generic case

$$
f_{x x}(0,0)<0
$$

the linearization of $(2.3)$ about $(0,0)$ has eigenvalues $\pm \lambda$ with

$$
\lambda=\left[-f_{x x}(0,0) f_{u u}(0,0)\right]^{1 / 2} .
$$

The asymptotic value of the period of a periodic orbit at the level $f(w, \xi)=-p<0$, with $p$ small, is

$$
T \sim \frac{2}{\lambda} \log \frac{1}{p} .
$$

The same number estimates twice the time it takes the orbit at the level $f(w, \xi)=p$ to traverse the interval $[0,1]$.

3. Weak solutions and generalized characteristics. We extend the domain of $\phi$ onto $(-\infty, \infty)$ by setting $\phi(x)=0$ for $x \notin(0,1)$. By standard theory (e.g. [6]), there exists a unique bounded measurable function $u(x, t)$ on $(-\infty, \infty) \times(0, \infty)$, such that, for any $t>0, u(\cdot, t)$ is of locally bounded variation on $(-\infty, \infty)$, which solves (in the sense of distributions) the Cauchy problem for (1.1), with initial datum $\phi$, and satisfies the "entropy" admissibility condition

$$
u(x+, t) \leqslant u(x-, t), \quad-\infty<x<\infty, \quad 0<t<\infty .
$$

We normalize $u$ by making it continuous from the left in $x$.

With $u(x, t)$ we associate generalized characteristics (cf. [2]), namely Lipschitz curves $\xi(\cdot)$ which satisfy almost everywhere the differential inclusion

$$
\dot{\xi}(t) \in\left[f_{u}(u(\xi(t)+, t), \xi(t)), f_{u}(u(\xi(t), t), \xi(t))\right]
$$


(compare with (2.1)). Every fixed point $(\bar{x}, \bar{t})$ of the upper half-plane is connected to the $x$-axis, $t=0$, by a set of backward characteristics that generally span a funnel confined between a minimal characteristic and a maximal characteristic. These extreme backward characteristics coincide if and only if $(\bar{x}, \bar{t})$ is a point of continuity for $u(x, t)$.

Let $\xi(\cdot)$ denote the minimal (or maximal) backward characteristic through $(\bar{x}, \bar{t})$. It is remarkable that $\xi(\cdot)$ is always a classical characteristic, in the following sense: along $\xi(\cdot)$, $u$ is continuous, i.e., $u(\xi(t)+, t)=u(\xi(t), t):=w(t), 0<t<\bar{t}$; the pair $(\xi(\cdot), w(\cdot))$ is the solution of $(2.3)$ on $(0, \bar{t})$, under the initial condition $\xi(\bar{t})=\bar{x}, w(\bar{t})=u(\xi(\bar{t}), \bar{t})$ (or $w(\bar{t})=u(\xi(\bar{t})+, \bar{t}))$. This property, established in [2], relies crucially upon assumption (1.5).

Let us now fix any $\bar{t}>0$ and let us consider the minimal backward characteristic $\xi(\cdot)$ through $(0, \bar{t})$. If $u(0, \bar{t})>0$ then it is easily seen from the phase portrait of orbits of (2.3), sketched in Fig. 1, that $\xi(t)<0$ and $u(\xi(t), t)>0$, for all $0 \leqslant t<\bar{t}$. However, this is a contradiction, since it should be $u(\xi(0), 0)=\phi(\xi(0))=0$. We have thus shown that $u(0, \bar{t}) \leqslant 0$ and a fortiori $f_{u}(u(0+, \bar{t}), 0) \leqslant 0$. A similar argument shows that $f_{u}(u(1-, \bar{t}), 1) \geqslant 0$, for any $\bar{t}>0$. Therefore, the restriction of $u(x, t)$ on the strip $(0,1) \times(0, \infty)$ satisfies the boundary conditions (1.3). In fact, using the characteristics it is easy to see that $u(x, t)$ is the only admissible solution of $(1.1),(1.2),(1.3)$. We collect our results in the following

Proposition 3.1. For any $\phi \in L^{\infty}(0,1)$ there exists a unique admissible solution of (1.1), (1.2), (1.3). Furthermore, every backward characteristic emanating from any point $(\bar{x}, \bar{t})$ of the strip $[0,1] \times(0, \infty)$ stays trapped inside the strip and it is intercepted, at $t=0$, by the $x$-axis at a point in the interval $[0,1]$.

4. Large time behavior of solutions. In this section we determine the asymptotic behavior, as $t \rightarrow \infty$, of the solution $u(x, t)$ of (1.1), (1.2), (1.3), in terms of the initial datum $\phi$.

LemMA 4.1. As $t \rightarrow \infty$,

$$
f(u(x, t), x) \rightarrow 0, \quad 0<x<1,
$$

uniformly on $(0,1)$.

Proof. Fix $t$ positive large, take any $x \in(0,1)$ and let $\xi(\cdot)$ denote the minimal backward characteristic through $(x, t)$.

Suppose first $f(u(x, t), x)>0$. Then $\xi(\cdot)$ is associated with one of the monotone orbits (cf. Fig. 1). The fact that $\xi(\cdot)$ sojourns in the interval $(0,1)$ over the long time $t$ implies that this orbit has to be close to one of the two heteroclinic orbits, i.e., $f(u(x, t), x)$ must be small.

Assume now $f(u(x, t), x)<0$, in which case $\xi(\cdot)$ is associated with one of the periodic orbits that surround the center $(a, 0)$ (cf. Fig. 1). Let $T$ be the period of this orbit. Suppose $T<t$. Consider the minimal backward characteristic $\zeta(\cdot)$ emanating from the point $(x-\eta, t)$, where $\eta>0$. When $\eta$ is sufficiently small, $\zeta(\cdot)$ is also associated with a periodic orbit either with period slightly lower than $T$ and amplitude slightly smaller than the amplitude of $\xi(\cdot)$ or with period slightly higher than $T$ and amplitude slightly bigger 
than the amplitude of $\xi(\cdot)$. In either case, it is easy to see that $\xi(\cdot)$ and $\zeta(\cdot)$ would have to intersect at least once in the interval $(0, t)$. However, this is impossible because, as stated in Section 3, minimal backward characteristics always pass through points of continuity of $u(x, t)$. It thus follows that $T \geqslant t$, i.e., the period of the orbit is large. But then the orbit must lie in the vicinity of the two heteroclinic orbits. In particular, $f(u(x, t), x)$ has to be near zero. This completes the proof.

The above result together with (2.5) and (3.1) suggests that, for $t$ large, $u(\cdot, t)$ is close to the supersonic steady state $v_{+}(\cdot)$ or to the subsonic steady state $v_{-}(\cdot)$ or to a discontinuous steady state that contains one shock connecting the supersonic to the subsonic branch. We proceed to determine the asymptotic profile and, in particular, the location of the shock.

On $[0,1]$ we define the continuous functions

$$
\left\{\begin{array}{l}
\Phi_{+}(x):=\int_{0}^{x}\left[\phi(y)-v_{+}(y)\right] d y, \\
\Phi_{-}(x):=\int_{0}^{x}\left[\phi(y)-v_{-}(y)\right] d y .
\end{array}\right.
$$

We let $x_{+}$(or $x_{-}$) denote the minimum (or maximum) of the set of points in $[0,1]$ at which $\Phi_{+}(\cdot)$ (or $\left.\Phi_{-}(\cdot)\right)$ attains its minimum over $[0,1]$. In particular, $\Phi_{+}\left(x_{+}\right) \leqslant \Phi_{+}\left(x_{-}\right)$ and $\Phi_{-}\left(x_{-}\right) \leqslant \Phi_{-}\left(x_{+}\right)$whence it follows easily

$$
\begin{gathered}
0 \leqslant x_{-} \leqslant x_{+} \leqslant 1, \\
\int_{x_{-}}^{x_{+}} v_{-}(y) d y \leqslant \int_{x_{-}}^{x_{+}} \phi(y) d y \leqslant \int_{x_{-}}^{x_{+}} v_{+}(y) d y .
\end{gathered}
$$

We seek $\bar{x}$ in $[0,1]$ such that

$$
\int_{0}^{\bar{x}} v_{+}(y) d y+\int_{\bar{x}}^{1} v_{-}(y) d y=\int_{0}^{x_{-}} v_{-}(y) d y+\int_{x_{-}}^{x_{+}} \phi(y) d y+\int_{x_{+}}^{1} v_{+}(y) d y .(4.5
$$

By virtue of (4.4), for any $\phi \in L^{\infty}(0,1)$ there is a unique $\bar{x}$ that satisfies (4.5). In particular, $\bar{x}=0$ if and only if $x_{-}=x_{+}=1$ and $\bar{x}=1$ if and only if $x_{-}=x_{+}=0$.

Theorem 4.1. Let $\bar{x} \in[0,1]$ be defined, as above, via (4.5). (a) If $\bar{x}=0$ then, as $t \rightarrow \infty$,

$$
u(x, t) \rightarrow v_{-}(x), \quad 0<x<1,
$$

uniformly on $(0,1)$. (b) If $\bar{x}=1$ then, as $t \rightarrow \infty$,

$$
u(x, t) \rightarrow v_{+}(x), \quad 0<x<1,
$$

uniformly on $(0,1)$. (c) If $\bar{x} \in(0,1)$ then, as $t \rightarrow \infty$,

$$
u(x, t) \rightarrow\left\{\begin{array}{ll}
v_{+}(x), & 0<x<\bar{x} \\
v_{-}(x), & \bar{x}<x<1
\end{array},\right.
$$

uniformly on $(0, \bar{x}-\eta) \cup(\bar{x}+\eta, 1)$, for any $\eta>0$.

Proof. We let $\left\{\xi_{-}(\cdot), w_{-}(\cdot)\right\}$ denote the solution on $[0, \infty)$ of the system (2.3) with initial conditions $\xi_{-}(0)=x_{-}, w_{-}(0)=v_{-}\left(x_{-}\right)$. If $x_{-}=0$, then $\xi_{-}(t) \equiv 0, w_{-}(t) \equiv 0$; if $x_{-}=1$, then $\xi_{-}(t) \equiv 1, w_{-}(t) \equiv 0$; if $x_{-} \in(0,1)$, then $\xi_{-}(\cdot)$ is a decreasing function that 
tends to zero as $t \rightarrow \infty$ and $w_{-}(t)=v_{-}\left(\xi_{-}(t)\right)$. Moreover,

$$
\dot{\xi}_{-}(t)=f_{u}\left(v_{-}\left(\xi_{-}(t)\right), \xi_{-}(t)\right), \quad 0<t<\infty .
$$

We fix $\bar{t}>0$ and we let $\xi(\cdot)$ denote the minimal backward characteristic of $u(x, t)$ emanating from the point $\left(\xi_{-}(\bar{t}), \bar{t}\right)$. We claim that

$$
\begin{aligned}
& \int_{0}^{i}\left\{f\left(u\left(\xi_{-}(t), t\right), \xi_{-}(t)\right)-f\left(v_{-}\left(\xi_{-}(t)\right), \xi_{-}(t)\right)\right. \\
& \left.\quad-f_{u}\left(v_{-}\left(\xi_{-}(t)\right), \xi_{-}(t)\right)\left[u\left(\xi_{-}(t), t\right)-v_{. .}\left(\xi_{-}(t)\right)\right]\right\} d t \\
& \quad+\int_{0}^{t}\left\{f\left(v_{-}(\xi(t)), \xi(t)\right)-f(u(\xi(t), t), \xi(t))\right. \\
& \left.-f_{u}(u(\xi(t), t), \xi(t))\left[v_{-}(\xi(t))-u(\xi(t), t)\right]\right\} d t \\
& \quad=\int_{\xi(0)}^{x_{-}}\left[\phi(y)-v_{-}(y)\right] d y=\Phi_{-}\left(x_{-}\right)-\Phi_{-}(\xi(0)) .
\end{aligned}
$$

To verify (4.8) we integrate (1.1) over the domain bordered by $\xi(\cdot), \xi_{-}(\cdot)$ and the $x$-axis, we apply the Gauss-Green theorem (applicable within the context of BV functions), and use (2.1), (4.7), (2.5), and (4.2). We now note that each one of the two integrals on the left-hand side of (4.8) is nonnegative, by virtue of (1.5). On the other hand, the right-hand side of (4.8) is nonpositive, by account of the selection of $x_{-}$. Therefore, all terms in (4.8) vanish. It follows that $\xi_{-}(\cdot)$ is a characteristic for $u(x, t)$ and

$$
u\left(\xi_{-}(t), t\right)=v_{-}\left(\xi_{-}(t)\right), \quad 0<t<\infty .
$$

Similarly, let $\left\{\xi_{+}(\cdot), w_{+}(\cdot)\right\}$ denote the solution of system $(2.3)$ on $[0, \infty)$ with initial conditions $\xi_{+}(0)=x_{+}, w_{+}(0)=v_{+}\left(x_{+}\right)$. When $x_{+}=0$, then $\xi_{+}(t) \equiv 0, w_{+}(t) \equiv 0$; when $x_{+}=1$, then $\xi_{+}(t) \equiv 1, w_{+}(t) \equiv 0$; when $x_{+} \in(0,1)$, then $\xi_{+}(\cdot)$ is an increasing function that tends to one as $t \rightarrow \infty$ and $w_{+}(t)=v_{+}\left(\xi_{+}(t)\right)$. Furthermore, it can be shown, as above, that $\xi_{+}(\cdot)$ is a characteristic for $u(x, t)$ and

$$
u\left(\xi_{+}(t), t\right)=v_{+}\left(\xi_{+}(t)\right), \quad 0<t<\infty .
$$

Let us first consider the nondegenerate case $x_{-}<1, x_{+}>0$ so that $\bar{x} \in(0,1)$. We fix $\bar{t}>0$, we integrate (1.1) over the domain $\left\{(x, t): 0<t<\bar{t}, \xi_{-}(t)<x<\xi_{+}(t)\right\}$ and we apply the Gauss-Green theorem. Using (4.9) and (4.10), this yields

$$
\begin{aligned}
\int_{\xi_{(}(i)}^{\xi_{+}(i)} u(x, \bar{t}) d x & -\int_{x}^{x_{+}} \phi(y) d y \\
+ & \int_{0}^{i}\left\{f\left(v_{+}\left(\xi_{+}(t)\right), \xi_{+}(t)\right)-\dot{\xi}_{+}(t) v_{+}\left(\xi_{+}(t)\right)\right\} d t \\
& -\int_{0}^{i}\left\{f\left(v_{-}\left(\xi_{-}(t)\right), \xi_{-}(t)\right)-\dot{\xi}_{-}(t) v_{-}\left(\xi_{-}(t)\right)\right\} d t=0 .
\end{aligned}
$$

Recalling (2.5), we may rewrite (4.11) in the form

$$
\int_{\xi_{-}(i)}^{\xi_{+}(\bar{t})} u(x, \bar{t}) d x=\int_{x}^{x_{+}} \phi(y) d y+\int_{x_{+}}^{\xi_{+}(i)} v_{+}(\xi) d \xi-\int_{x_{-}}^{\xi_{(i)}} v_{-}(\xi) d \xi .
$$

As $\bar{t} \rightarrow \infty, \xi_{-}(\bar{t}) \rightarrow 0$ and $\xi_{+}(\bar{t}) \rightarrow 1$. Therefore, combining (4.12) with (3.1), (4.1), and (4.5) we arrive at $(4.6)_{\text {. }}$. 
Next consider the degenerate case $x_{-}=x_{+}=0$ so that $\bar{x}=1$. Then $\xi_{-}(t)=\xi_{+}(t)=0$ and $u(0, t)=0,0<t<\infty$. We fix $\bar{t}>0$ and let $\xi(\cdot)$ denote the minimal backward characteristic that emanates from the point $(1, \bar{t})$. We claim that

$$
\begin{aligned}
& \int_{0}^{1}\left[u(x, \bar{t})-v_{+}(x)\right] d x-\int_{0}^{\xi(0)}\left[\phi(y)-v_{+}(y)\right] d y \\
& +\int_{0}^{i}\left\{f(u(\xi(t), t), \xi(t))-f\left(v_{+}(\xi(t)), \xi(t)\right)\right. \\
& \left.\quad-f_{u}(u(\xi(t), t), \xi(t))\left[u(\xi(t), t)-v_{+}(\xi(t))\right]\right\} d t=0 .
\end{aligned}
$$

To verify (4.13) integrate (1.1) over the domain $\{(x, t): 0<t<\bar{t}, 0<x<\xi(t)\}$, apply the Gauss-Green theorem and use (2.1) and (2.5). We note that the second integral in (4.13) is nonnegative, because $x_{+}=0$. Moreover, the third integral in (4.13) is nonpositive, by virtue of (1.5). It follows that the first integral in (4.13) is nonnegative and this, in conjunction with (4.1), yields $(4.6)_{\mathrm{b}}$.

A similar argument applies to the remaining degenerate case $x_{-}=x_{+}=1$ (so that $\bar{x}=0)$ and establishes (4.6) $)_{\mathrm{a}}$. This completes the proof of the theorem.

More detailed analysis yields precise decay rates to the steady state. In the generic case (2.6) and by virtue of (2.8) the argument in the proof of Lemma 4.1 now gives

$$
f(u(x, t), x)=O\left(e^{-\lambda t / 2}\right) .
$$

Consequently, the conclusion of Theorem 4.1, Eq. (4.6), is strengthened as follows: As $t \rightarrow \infty$,

$$
\begin{aligned}
& u(x, t)=v_{-}(x)+O\left(e^{-\lambda t / 4}\right), 0<x<1 \quad \text { if } \bar{x}=0, \\
& u(x, t)=v_{+}(x)+O\left(e^{-\lambda t / 4}\right), 0<x<1 \quad \text { if } \bar{x}=1 \text {, } \\
& u(x, t)=\left\{\begin{array}{ll}
v_{+}(x)+O\left(e^{-\lambda t / 4}\right), & 0<x<X(t) \\
v_{-}(x)+O\left(e^{-\lambda t / 4}\right), & X(t)<x<1
\end{array} \quad \text { if } 0<\bar{x}<1,\right.
\end{aligned}
$$

where

$$
X(t)=\bar{x}+O\left(e^{-\lambda t / 4}\right)
$$

\section{REFERENCES}

[1] C. M. Dafermos, Characteristics in hyperbolic conservation laws, Nonlinear Analysis and Mechanics (R. J. Knops, Ed.), Research Notes in Math. No. 17, Pitman, London pp. 1-58, 1977

[2] C. M. Dafermos, Generalized characteristics and the structure of solutions of hyperbolic conservation laws, Indiana Univ. Math. J. 26, 1097-1119 (1977)

[3] C. M. Dafermos, Large time behavior of solutions of hyperbolic halance laws, Bull. Greek Math. Soc. 25, 15-29 (1984)

[4] C. M. Dafermos, Regularity and large time behavior of solutions of a conservation law without convexity, Proc. Royal Soc. Edinburgh 99A, 201-239 (1985)

[5] G. Kreiss, and H. O. Kreiss, Convergence to steady state of solutions of Burgers' equation, Appl. Num. Math. 2, 161-179 (1986)

[6] O. A. Oleinik, Discontinuous solutions of nonlinear differential equations, Uspehi Mat. Nauk (N.S.) 12, 3-73 (1957). AMS Transl. Series 2, Vol. 26, pp. 95-172

[7] M. D. Salas, S. Abarbanel, and D. Gottlieb, Multiple steady states for characteristic initial value problems, Appl. Num. Math. 2, 193-210 (1986). 